\title{
Oscillation Theorems for Even Order Damped Equations with Distributed Deviating Arguments
}

\author{
Chunxia Gao and Peiguang Wang \\ College of Electronic and Information Engineering, Hebei University, Baoding 071002, China \\ Correspondence should be addressed to Peiguang Wang; pgwang@mail.hbu.edu.cn
}

Received 24 August 2013; Accepted 8 November 2013

Academic Editor: Miroslava Růžičková

Copyright (C) 2013 C. Gao and P. Wang. This is an open access article distributed under the Creative Commons Attribution License, which permits unrestricted use, distribution, and reproduction in any medium, provided the original work is properly cited.

A class of even order damped differential equations with distributed deviating arguments are investigated. Several new criteria that ensure the oscillation of solutions are obtained. To demonstrate the validity of the results obtained, two examples are given.

\section{Introduction and Lemmas}

Oscillatory behavior of solutions for different types of second-order differential equations with damping has been widely discussed by using different techniques. Here, we particularly refer the reader to the papers [1-9] and the references quoted therein. However, very little is known for the case of higher order damped functional differential equations with deviating arguments, especially the case with distributed deviating arguments. In this paper, we deal with the following class of even order functional differential equations with damping:

$$
\begin{aligned}
x^{(n)}(t)+p(t) x^{(n-1)}(t) & \\
+\int_{\alpha}^{\beta} q(t, \xi) f\left(x\left[g_{1}(t, \xi)\right], \ldots, x\left[g_{m}(t, \xi)\right]\right) d \mu(\xi) & =0, \\
t \geq t_{0} & >0 .
\end{aligned}
$$

Our aim is to get the criteria for the oscillatory solutions of (1).

Throughout this paper, we assume that the following conditions hold:

$\left(\mathrm{H}_{1}\right) n$ is an even positive integer;

$\left(\mathrm{H}_{2}\right) p(t) \in C\left(\left[t_{0}, \infty\right), R_{+}\right), q(t, \xi) \in C\left(\left[t_{0}, \infty\right) \times[\alpha, \beta], R_{+}\right)$ is not identically zero on any $[T, \infty) \times[\alpha, \beta]$ for $T \geq t_{0}$, and

$$
\lim _{t \rightarrow \infty} \int_{t_{1}}^{t} \exp \left(-\int_{t_{1}}^{s} p(\tau) d \tau\right) d s=\infty, \quad t_{1} \geq t_{0}
$$

$\left(\mathrm{H}_{3}\right) f\left(u_{1}, u_{2}, \ldots, u_{m}\right) \in C\left(R^{m}, R\right)$ has the same sign as $u_{1}, u_{2}, \ldots, u_{m}$ when $u_{1}, u_{2}, \ldots, u_{m}$ have the same sign, $g_{i}(t, \xi) \in C\left(\left[t_{0}, \infty\right) \times[\alpha, \beta], R_{+}\right), \mu(\xi) \in([\alpha, \beta], R)$ is nondecreasing, and the integral of (1) is a Stieltjes one.

In the sequel, it will be always assumed that solutions of (1) exist for any $t_{0} \geq 0$. A solution $x(t)$ of (1) is called eventually positive solution (or negative solution) if there exists a sufficiently large positive number $t_{1} \geq t_{0}$, such that $x(t)>0($ or $x(t)<0)$ for all $t \geq t_{1}$. A nontrivial solution $x(t)$ of (1) is called oscillatory if it has arbitrary large zeros; otherwise it is called nonoscillatory. Equation (1) is called oscillatory if all its solutions are oscillatory.

Remark 1. Since the integral of (1) is a Stieltjes one, it includes the following equations:

$$
\begin{aligned}
x^{(n)}(t)+p(t) x^{(n-1)}(t) & \\
+\sum_{i=1}^{m} q_{i}(t) f\left(x\left[g_{1}(t)\right], \ldots, x\left[g_{m}(t)\right]\right) & =0, \\
t \geq t_{0} & >0 .
\end{aligned}
$$

The following lemmas will be useful to the proof of the main results to be presented in this paper. 
Lemma 2 (see [10]). Let $u(t)$ be a positive and $n$ times differentiable function on $R_{+}$. If $u^{(n)}(t)$ is of constant sign and not identically zero on any ray $\left[t_{1},+\infty\right)$ for $t_{1}>0$, then there exists $a t_{u} \geq t_{1}$ and an integer $l(0 \leq l \leq n)$, with $n+l$ even for $u(t) u^{(n)}(t) \geq 0$ or $n+l$ odd for $u(t) u^{(n)}(t) \leq 0$; and for $t \geq t_{u}$,

$$
\begin{gathered}
u(t) u^{(k)}(t)>0, \quad 0 \leq k \leq l ; \\
(-1)^{k-l} u(t) u^{(k)}(t)>0, \quad l \leq k \leq n .
\end{gathered}
$$

Lemma 3 (see [11]). Suppose that the conditions of Lemma 2 are satisfied, and

$$
u^{(n-1)}(t) u^{(n)}(t) \leq 0, \quad t \geq t_{u},
$$

then there exists a constant $\theta \in(0,1)$ such that for sufficiently large $t$, there exists a constant $M_{\theta}>0$ satisfying

$$
\left|u^{\prime}\left(\frac{t}{2}\right)\right| \geq M_{\theta} t^{n-2}\left|u^{(n-1)}(t)\right| .
$$

We say that a function $H=H(t, s)$ belongs to a function class $\Phi$, denoted by $H \in \Phi$, if $H \in C\left(D, R_{+}\right)$, where $D=$ $\{(t, s):-\infty<s \leq t<\infty\}$, satisfies

(i) $H(t, t)=0$, for $t \geq t_{0}$ and $H(t, s)>0$, for $t>s \geq t_{0}$;

(ii) partial derivatives $\partial H / \partial t$ and $\partial H / \partial s$ exist, and

$$
\begin{gathered}
\frac{\partial H}{\partial t}=h_{1}(t, s) \sqrt{H(t, s)}, \\
\frac{\partial H}{\partial s}=-h_{2}(t, s) \sqrt{H(t, s)},
\end{gathered}
$$

where $h_{1}, h_{2} \in L_{\text {loc }}(D, R)$.

\section{Oscillation Results for $f\left(u_{1}, \ldots, u_{m}\right)$ with Monotonicity}

Throughout this section, we assume that the following conditions hold.

$\left(\mathrm{A}_{1}\right)$ There exist functions $\sigma_{i}(t) \in C^{\prime}\left(\left[t_{0}, \infty\right),(0, \infty)\right)$, such that $\sigma_{i}(t)=\min \left\{t, \inf _{\xi \in[\alpha, \beta]} g_{i}(t, \xi)\right\}, \lim _{t \rightarrow \infty}$ $\sigma_{i}(t)=\infty, \sigma_{i}^{\prime}(t)>0$, and $i=1,2, \ldots, m$.

$\left(\mathrm{A}_{2}\right)\left(\partial / \partial u_{i}\right) f\left(u_{1}, \ldots, u_{m}\right) \equiv f_{i}^{\prime}\left(u_{1}, \ldots, u_{m}\right)$ exists, and $f_{i}^{\prime}\left(u_{1}, \ldots, u_{m}\right) \geq \lambda_{i}>0$ for $u_{i} \neq 0, i=1,2, \ldots, m$, where $\lambda_{i}>0$ are some constants, and $i=1,2, \ldots, m$.

Lemma 4. Let $x(t)$ be an eventually positive solution of (1). Then, there exists a sufficiently large $T_{0} \geq t_{0}$, such that for all $t \geq T_{0}$

$$
x^{\prime}(t)>0, \quad x^{(n-1)}(t)>0, \quad x^{(n)}(t) \leq 0 .
$$

Proof. From the assumption, there exists a sufficiently large $t_{1} \geq t_{0}$, such that $x(t)>0$ for $t \geq t_{1}$. Further from $\left(\mathrm{A}_{1}\right)$, there exists $t_{2} \geq t_{1}$ such that for all $t \geq t_{2}$

$$
\begin{array}{r}
\sigma_{i}(t) \geq t_{1}, \quad g_{i}(t, \xi) \geq \sigma_{i}(t) \geq t_{1}, \\
i=1,2, \ldots, m ; \quad \xi \in[\alpha, \beta] .
\end{array}
$$

Hence, for all $t \geq t_{2}$

$$
\begin{aligned}
x\left[\sigma_{i}(t)\right] & >0, \quad x\left[g_{i}(t, \xi)\right]>0, \\
i & =1,2, \ldots, m ; \xi \in[\alpha, \beta],
\end{aligned}
$$

and from $\left(\mathrm{H}_{3}\right)$, we have for all $t \geq t_{2}$ and $\xi \in[\alpha, \beta]$

$$
\begin{gathered}
f\left(x\left[\sigma_{1}(t)\right], \ldots, x\left[\sigma_{m}(t)\right]\right)>0, \\
f\left(x\left[g_{1}(t, \xi)\right], \ldots, x\left[g_{m}(t, \xi)\right]\right)>0 .
\end{gathered}
$$

Let

$$
v(t)=\exp \int_{t_{2}}^{t} p(s) d s, \quad w(t)=x^{(n-1)}(t) v(t), \quad t \geq t_{2},
$$

then it is easy to know that

$$
\begin{aligned}
& w^{\prime}(t)=\left(x^{(n)}(t)+p(t) x^{(n-1)}(t)\right) v(t) \\
&=-\int_{\alpha}^{\beta} q(t, \xi) f\left(x\left[g_{1}(t, \xi)\right], \ldots, x\left[g_{m}(t, \xi)\right]\right) \\
& \quad \times d \mu(\xi) v(t) \leq 0,
\end{aligned}
$$

which implies that $w(t)$ is nonincreasing on $\left[t_{2},+\infty\right)$.

Now, we claim that $x^{(n-1)}(t) \geq 0, t \geq t_{2}$. Otherwise, there exists $t_{3} \geq t_{2}$ such that $x^{(n-1)}\left(t_{3}\right)<0$. Therefore,

$$
\begin{gathered}
x^{(n-1)}(t) v(t) \leq x^{(n-1)}\left(t_{3}\right) v\left(t_{3}\right), \quad t \geq t_{3}, \\
\int_{t_{3}}^{t} x^{(n-1)}(\tau) d \tau \leq x^{(n-1)}\left(t_{3}\right) v\left(t_{3}\right) \int_{t_{3}}^{t} \frac{1}{v(\tau)} d \tau, \quad t \geq t_{3}, \\
x^{(n-2)}(t) \leq x^{(n-2)}\left(t_{3}\right)+x^{(n-1)}\left(t_{3}\right) v\left(t_{3}\right) \int_{t_{3}}^{t} \frac{1}{v(\tau)} d \tau, \\
t \geq t_{3} .
\end{gathered}
$$

Using $\left(\mathrm{H}_{2}\right)$, we see that $\lim _{t \rightarrow+\infty} x^{(n-2)}(t)=-\infty$. Ulteriorly, we can prove $\lim _{t \rightarrow+\infty} x(t)=-\infty$, which contradicts $x(t)>$ $0, t \geq t_{1}$.

Furthermore, from (1), for all $t \geq t_{2}$, we have

$$
\begin{aligned}
x^{(n)}(t)= & -p(t) x^{(n-1)}(t) \\
& -\int_{\alpha}^{\beta} q(t, \xi) f\left(x\left[g_{1}(t, \xi)\right], \ldots, x\left[g_{m}(t, \xi)\right]\right) \\
& \times d \mu(\xi) \leq 0 .
\end{aligned}
$$

Thus, from Lemma 2, there exist $T_{0} \geq t_{2}$ and an odd number $l(0<l<n)$, such that for $t \geq T_{0}$, we have

$$
\begin{aligned}
& x^{(k)}(t)>0, \quad 0 \leq k \leq l ; \\
& (-1)^{k-l} x^{(k)}(t)>0, \quad l \leq k \leq n .
\end{aligned}
$$

By choosing $k=1$ and $n-1$, we have $x^{\prime}(t)>0$ and $x^{(n-1)}(t)>$ 0 for $t \geq T_{0}$. The proof is completed. 
Lemma 5. Let $x(t)$ be an eventually positive solution of (1). Then, there exists a sufficiently large $T_{0} \geq t_{0}$, such that for any interval $[c, b) \subset\left[T_{0}, \infty\right)$, if let

$$
y(t)=\frac{\rho(t) x^{(n-1)}(t)}{f\left(x\left[\sigma_{1}(t) / 2\right], \ldots, x\left[\sigma_{m}(t) / 2\right]\right)}, \quad t \in[c, b),
$$

where $\rho(t) \in C^{\prime}\left(\left[t_{0}, \infty\right),(0, \infty)\right)$, then for any $H \in \Phi$,

$$
\begin{aligned}
\int_{c}^{b} H(b, s) \rho(s)\left(\int_{\alpha}^{\beta} q(s, \xi) d \mu(\xi)\right) d s \\
\leq H(b, c) y(c)+\frac{1}{2} \int_{c}^{b} \frac{\rho(s)}{\sum_{i=1}^{m} \lambda_{i} M_{\theta} \sigma_{i}^{n-2}(s) \sigma_{i}^{\prime}(s)} \\
\quad \times\left[h_{2}(b, s)-\sqrt{H(b, s)}\left(\frac{\rho^{\prime}(s)}{\rho(s)}-p(s)\right)\right]^{2} d s .
\end{aligned}
$$

Proof. From (1) and (16), we have that for $t \in[c, b)$,

$$
\begin{aligned}
y^{\prime}(t) & \\
= & \frac{\rho(t) x^{(n)}(t)+\rho^{\prime}(t) x^{(n-1)}(t)}{f\left(x\left[\sigma_{1}(t) / 2\right], \ldots, x\left[\sigma_{m}(t) / 2\right]\right)} \\
& -\frac{y(t)}{f\left(x\left[\sigma_{1}(t) / 2\right], \ldots, x\left[\sigma_{m}(t) / 2\right]\right)} \\
& \times\left(\frac { 1 } { 2 } \sum _ { i = 1 } ^ { m } f _ { i } ^ { \prime } \left(x\left[\frac{\sigma_{1}(t)}{2}\right], \ldots,\right.\right. \\
= & -\rho(t) \frac{\int_{\alpha}^{\beta} q(t, \xi) f\left(x\left[g_{1}(t, \xi)\right], \ldots, x\left[g_{m}(t, \xi)\right]\right) d \mu(\xi)}{f\left(x\left[\sigma_{1}(t) / 2\right], \ldots, x\left[\sigma_{m}(t) / 2\right]\right)} \\
& +\left(\frac{\rho^{\prime}(t)}{\rho(t)}-p(t)\right) y(t) \\
& -\frac{y(t)}{f\left(x\left[\sigma_{1}(t) / 2\right], \ldots, x\left[\sigma_{m}(t) / 2\right]\right)} \\
& \times\left(\frac{1}{2} \sum_{i=1}^{m} f_{i}^{\prime}\left(x\left[\frac{\sigma_{1}(t)}{2}\right], \ldots, x\left[\frac{\sigma_{m}(t)}{2}\right]\right)\right. \\
& \left.\times x^{\prime}\left[\frac{\sigma_{i}(t)}{2}\right] \sigma_{i}^{\prime}(t)\right) .
\end{aligned}
$$

From Lemma 4, there exists a sufficiently large $T_{0} \geq t_{0}$ such that $x^{\prime}(t)>0$ and $x^{(n)}(t) \leq 0$ for $t \geq T_{0}$. Further from $\left(\mathrm{A}_{1}\right)$, for all $t \geq T_{0}$

$$
\begin{array}{r}
\sigma_{i}(t) \leq t, \quad g_{i}(t, \xi) \geq \sigma_{i}(t) \geq \frac{\sigma_{i}(t)}{2}, \\
i=1,2, \ldots, m ; \xi \in[\alpha, \beta] .
\end{array}
$$

Hence, for all $t \geq T_{0}$, we have

$$
\begin{array}{r}
x\left[g_{i}(t, \xi)\right] \geq x\left[\frac{\sigma_{i}(t)}{2}\right], \quad x^{(n-1)}\left[\sigma_{i}(t)\right] \geq x^{(n-1)}(t), \\
i=1,2, \ldots, m ; \xi \in[\alpha, \beta] .
\end{array}
$$

In view of (20) and $\left(A_{2}\right)$, for all $t \geq T_{0}$

$$
\begin{aligned}
f & \left(x\left[g_{1}(t, \xi)\right], \ldots, x\left[g_{m}(t, \xi)\right]\right) \\
& \geq f\left(x\left[\frac{\sigma_{1}(t)}{2}\right], \ldots, x\left[\frac{\sigma_{m}(t)}{2}\right]\right), \quad \xi \in[\alpha, \beta] .
\end{aligned}
$$

Thus, for all $t \geq T_{0}$

$$
\begin{aligned}
\frac{\int_{\alpha}^{\beta} q(t, \xi) f\left(x\left[g_{1}(t, \xi)\right], \ldots, x\left[g_{m}(t, \xi)\right]\right) d \mu(\xi)}{f\left(x\left[\sigma_{1}(t) / 2\right], \ldots, x\left[\sigma_{m}(t) / 2\right]\right)} \\
\quad \geq \int_{\alpha}^{\beta} q(t, \xi) d \mu(\xi) .
\end{aligned}
$$

Therefore, from (18)-(22) and Lemma 3, we obtain

$$
\begin{aligned}
y^{\prime}(t) \leq & -\rho(t) \int_{\alpha}^{\beta} q(t, \xi) d \mu(\xi)+\left(\frac{\rho^{\prime}(t)}{\rho(t)}-p(t)\right) y(t) \\
& -\frac{x^{(n-1)}(t)}{f\left(x\left[\sigma_{1}(t) / 2\right], \ldots, x\left[\sigma_{m}(t) / 2\right]\right)} \\
& \times\left(\frac{1}{2} \sum_{i=1}^{m} \lambda_{i} M_{\theta} \sigma_{i}^{n-2}(t) \sigma_{i}^{\prime}(t)\right) y(t) \\
= & -\rho(t) \int_{\alpha}^{\beta} q(t, \xi) d \mu(\xi)+\left(\frac{\rho^{\prime}(t)}{\rho(t)}-p(t)\right) y(t) \\
& -\rho^{-1}(t)\left(\frac{1}{2} \sum_{i=1}^{m} \lambda_{i} M_{\theta} \sigma_{i}^{n-2}(t) \sigma_{i}^{\prime}(t)\right) y^{2}(t)
\end{aligned}
$$

for all $t \geq T_{0}$.

Multiplying (23) by $H(t, s)$, then integrating it with respect to $s$ from $c$ to $t$ for $t \in[c, b)$ and using (i) and (ii), we get that

$$
\begin{aligned}
& \int_{c}^{t} H(t, s) \rho(s)\left(\int_{\alpha}^{\beta} q(s, \xi) d \mu(\xi)\right) d s \\
& \leq-\int_{c}^{t} H(t, s) y^{\prime}(s) d s \\
& \quad+\int_{c}^{t} H(t, s)\left(\frac{\rho^{\prime}(s)}{\rho(s)}-p(s)\right) y(s) d s \\
& \quad-\int_{c}^{t} H(t, s) \rho^{-1}(s)\left(\frac{1}{2} \sum_{i=1}^{m} \lambda_{i} M_{\theta} \sigma_{i}^{n-2}(s) \sigma_{i}^{\prime}(s)\right) y^{2}(s) d s
\end{aligned}
$$




$$
\begin{array}{rlr}
= & H(t, c) y(c)+\int_{c}^{t} \frac{\partial H(t, s)}{\partial s} y(s) d s & +\frac{1}{2} \int_{a}^{c} \frac{\rho(s)}{\sum_{i=1}^{m} \lambda_{i} M_{\theta} \sigma^{n-2}(s) \sigma_{i}^{\prime}(s)} \\
& +\int_{c}^{t} H(t, s)\left(\frac{\rho^{\prime}(s)}{\rho(s)}-p(s)\right) y(s) d s & \times\left[h_{1}(s, a)+\sqrt{H(s, a)}\left(\frac{\rho^{\prime}(s)}{\rho(s)}-p(s)\right)\right]^{2} d s . \\
& -\int_{c}^{t} H(t, s) \rho^{-1}(s)\left(\frac{1}{2} \sum_{i=1}^{m} \lambda_{i} M_{\theta} \sigma_{i}^{n-2}(s) \sigma_{i}^{\prime}(s)\right) y^{2}(s) d s & \text { Proof. Similar to the proof of Lemma 5, by multiplying (23) }
\end{array}
$$$$
=H(t, c) y(c)-\int_{c}^{t} \sqrt{H(t, s)}
$$$$
\times\left[h_{2}(t, s)-\sqrt{H(t, s)}\left(\frac{\rho^{\prime}(s)}{\rho(s)}-p(s)\right)\right] y(s) d s
$$$$
-\int_{c}^{t} H(t, s) \rho^{-1}(s)\left(\frac{1}{2} \sum_{i=1}^{m} \lambda_{i} M_{\theta} \sigma_{i}^{n-2}(s) \sigma_{i}^{\prime}(s)\right) y^{2}(s) d s
$$$$
=H(t, c) y(c)
$$$$
+\frac{1}{2} \int_{c}^{t} \frac{\rho(s)}{\sum_{i=1}^{m} \lambda_{i} M_{\theta} \sigma_{i}^{n-2}(s) \sigma_{i}^{\prime}(s)}
$$$$
\times\left[h_{2}(t, s)-\sqrt{H(t, s)}\left(\frac{\rho^{\prime}(s)}{\rho(s)}-p(s)\right)\right]^{2} d s
$$$$
-\int_{c}^{t}\left[\sqrt{\frac{H(t, s)}{\rho(s)}\left(\frac{1}{2} \sum_{i=1}^{m} \lambda_{i} M_{\theta} \sigma_{i}^{n-2}(s) \sigma_{i}^{\prime}(s)\right)} y(s)\right.
$$$$
\left.+\frac{h_{2}(t, s)-\sqrt{H(t, s)}\left(\left(\rho^{\prime}(s) / \rho(s)\right)-p(s)\right)}{\sqrt{2 \rho^{-1}(s)\left(\sum_{i=1}^{m} \lambda_{i} M_{\theta} \sigma_{i}^{n-2}(s) \sigma_{i}^{\prime}(s)\right)}}\right]^{2} d s
$$$$
\leq H(t, c) y(c)
$$$$
+\frac{1}{2} \int_{c}^{t} \frac{\rho(s)}{\sum_{i=1}^{m} \lambda_{i} M_{\theta} \sigma_{i}^{n-2}(s) \sigma_{i}^{\prime}(s)}
$$$$
\times\left[h_{2}(t, s)-\sqrt{H(t, s)}\left(\frac{\rho^{\prime}(s)}{\rho(s)}-p(s)\right)\right]^{2} d s .
$$

Proof. Similar to the proof of Lemma 5, by multiplying (23) by $H(s, t)$, then integrating it with respect to $s$ from $t$ to $c$ for $t \in(a, c]$, and then using (i) and (ii), we get that

$$
\begin{aligned}
& \int_{t}^{c} H(s, t) \rho(s)\left(\int_{\alpha}^{\beta} q(s, \xi) d \mu(\xi)\right) d s \\
& \leq-\int_{t}^{c} H(s, t) y^{\prime}(s) d s \\
& +\int_{t}^{c} H(s, t)\left(\frac{\rho^{\prime}(s)}{\rho(s)}-p(s)\right) y(s) d s \\
& -\int_{t}^{c} H(s, t) \rho^{-1}(s)\left(\frac{1}{2} \sum_{i=1}^{m} \lambda_{i} M_{\theta} \sigma_{i}^{n-2}(s) \sigma_{i}^{\prime}(s)\right) y^{2}(s) d s \\
& =-H(c, t) y(c)+\int_{t}^{c} \frac{\partial H(s, t)}{\partial s} y(s) d s \\
& +\int_{t}^{c} H(s, t)\left(\frac{\rho^{\prime}(s)}{\rho(s)}-p(s)\right) y(s) d s \\
& -\int_{t}^{c} H(s, t) \rho^{-1}(s)\left(\frac{1}{2} \sum_{i=1}^{m} \lambda_{i} M_{\theta} \sigma_{i}^{n-2}(s) \sigma_{i}^{\prime}(s)\right) y^{2}(s) d s \\
& =-H(c, t) y(c) \\
& +\int_{t}^{c} \sqrt{H(s, t)}\left[h_{1}(s, t)\right. \\
& \left.+\sqrt{H(s, t)}\left(\frac{\rho^{\prime}(s)}{\rho(s)}-p(s)\right)\right] y(s) d s \\
& -\int_{t}^{c} H(s, t) \rho^{-1}(s)\left(\frac{1}{2} \sum_{i=1}^{m} \lambda_{i} M_{\theta} \sigma_{i}^{n-2}(s) \sigma_{i}^{\prime}(s)\right) y^{2}(s) d s \\
& =-H(c, t) y(c)+\frac{1}{2} \int_{t}^{c} \frac{\rho(s)}{\sum_{i=1}^{m} \lambda_{i} M_{\theta} \sigma_{i}^{n-2}(s) \sigma_{i}^{\prime}(s)} \\
& \times\left[h_{1}(s, t)+\sqrt{H(s, t)}\left(\frac{\rho^{\prime}(s)}{\rho(s)}-p(s)\right)\right]^{2} d s \\
& -\int_{t}^{c}\left[\sqrt{\frac{H(s, t)}{\rho(s)}\left(\frac{1}{2} \sum_{i=1}^{m} \lambda_{i} M_{\theta} \sigma_{i}^{n-2}(s) \sigma_{i}^{\prime}(s)\right)} y(s)\right. \\
& \left.-\frac{h_{1}(s, t)+\sqrt{H(s, t)}\left(\left(\rho^{\prime}(s) / \rho(s)\right)-p(s)\right)}{\sqrt{2 \rho^{-1}(s)\left(\sum_{i=1}^{m} \lambda_{i} M_{\theta} \sigma_{i}^{n-2}(s) \sigma_{i}^{\prime}(s)\right)}}\right]^{2} d s
\end{aligned}
$$

Letting $t \rightarrow b^{-}$in the above, we obtain (17). The proof is completed.

Lemma 6. Let $x(t)$ be an eventually positive solution of (1). Then, there exists a sufficiently large $T_{0} \geq t_{0}$ such that for any interval $(a, c] \subset\left[T_{0}, \infty\right)$, if let $y(t)$ be defined by $(16)$ on $(a, c]$, then for any $H \in \Phi$,

$$
\begin{aligned}
& \int_{a}^{c} H(s, a) \rho(s)\left(\int_{\alpha}^{\beta} q(s, \xi) d \mu(\xi)\right) d s \\
& \quad \leq-H(c, a) y(c)
\end{aligned}
$$




$$
\begin{aligned}
\leq & -H(c, t) y(c)+\frac{1}{2} \int_{t}^{c} \frac{\rho(s)}{\sum_{i=1}^{m} \lambda_{i} M_{\theta} \sigma_{i}^{n-2}(s) \sigma_{i}^{\prime}(s)} \\
& \times\left[h_{1}(s, t)+\sqrt{H(s, t)}\left(\frac{\rho^{\prime}(s)}{\rho(s)}-p(s)\right)\right]^{2} d s .
\end{aligned}
$$

Letting $t \rightarrow a^{+}$in the above, we obtain (25). The proof is completed.

The following theorem is an immediate result from Lemmas 5 and 6.

Theorem 7. Assume that for each $T \geq t_{0}$ there exist $H \in \Phi$, $\rho \in C^{\prime}\left(\left[t_{0}, \infty\right),(0, \infty)\right)$ and $a, b, c \in R$, such that $T \leq a<c<$ $b$ and

$$
\begin{aligned}
\frac{1}{H(c, a)} \int_{a}^{c} H(s, a) \rho(s)\left(\int_{\alpha}^{\beta} q(s, \xi) d \mu(\xi)\right) d s \\
+\frac{1}{H(b, c)} \int_{c}^{b} H(b, s) \rho(s)\left(\int_{\alpha}^{\beta} q(s, \xi) d \mu(\xi)\right) d s \\
>\frac{1}{2}\left\{\frac{1}{H(c, a)} \int_{a}^{c} \frac{\rho(s)}{\sum_{i=1}^{m} \lambda_{i} M_{\theta} \sigma_{i}^{n-2}(s) \sigma_{i}^{\prime}(s)}\right. \\
\times\left[h_{1}(s, a)+\sqrt{H(s, a)}\left(\frac{\rho^{\prime}(s)}{\rho(s)}-p(s)\right)\right]^{2} d s \\
+\frac{1}{H(b, c)} \\
\times \int_{c}^{b} \frac{\rho(s)}{\sum_{i=1}^{m} \lambda_{i} M_{\theta} \sigma_{i}^{n-2}(s) \sigma_{i}^{\prime}(s)} \\
\left.\quad \times\left[h_{2}(b, s)-\sqrt{H(b, s)}\left(\frac{\rho^{\prime}(s)}{\rho(s)}-p(s)\right)\right]^{2} d s\right\} .
\end{aligned}
$$

Then (1) is oscillatory.

Proof. Suppose that (1) has a nonoscillatory solution $x(t)$. Without loss of generality, we assume that $x(t)$ is an eventually positive solution of (1). Then from Lemmas 5 and 6 , there exists a sufficiently large $T_{0} \geq t_{0}$, such that for any $(a, b) \subset\left[T_{0}, \infty\right)$, and for any $c \in(a, b), H \in \Phi$ and $\rho \in C^{\prime}\left(\left[t_{0}, \infty\right),(0, \infty)\right),(17)$ and $(25)$ hold. By dividing (17) and $(25)$ by $H(b, c)$ and $H(c, a)$, respectively, and then adding them, we have

$$
\begin{aligned}
& \frac{1}{H(c, a)} \int_{a}^{c} H(s, a) \rho(s)\left(\int_{\alpha}^{\beta} q(s, \xi) d \mu(\xi)\right) d s \\
& \quad+\frac{1}{H(b, c)} \int_{c}^{b} H(b, s) \rho(s)\left(\int_{\alpha}^{\beta} q(s, \xi) d \mu(\xi)\right) d s \\
& \quad \leq \frac{1}{2}\left\{\frac{1}{H(c, a)} \int_{a}^{c} \frac{\rho(s)}{\sum_{i=1}^{m} \lambda_{i} M_{\theta} \sigma_{i}^{n-2}(s) \sigma_{i}^{\prime}(s)}\right.
\end{aligned}
$$

$$
\begin{gathered}
\times\left[h_{1}(s, a)+\sqrt{H(s, a)}\left(\frac{\rho^{\prime}(s)}{\rho(s)}-p(s)\right)\right]^{2} d s \\
+\frac{1}{H(b, c)} \\
\times \int_{c}^{b} \frac{\rho(s)}{\sum_{i=1}^{m} \lambda_{i} M_{\theta} \sigma_{i}^{n-2}(s) \sigma_{i}^{\prime}(s)} \\
\times\left[h_{2}(b, s)-\sqrt{H(b, s)}\right. \\
\left.\left.\times\left(\frac{\rho^{\prime}(s)}{\rho(s)}-p(s)\right)\right]^{2} d s\right\},
\end{gathered}
$$

which contradicts the assumption (27) and completes the proof.

Theorem 8. Assume that for some $H \in \Phi, \rho \in C^{\prime}\left(\left[t_{0}, \infty\right)\right.$, $(0, \infty))$ and for each $r \geq t_{0}$,

$$
\begin{aligned}
\limsup _{t \rightarrow \infty} \int_{r}^{t}\left\{H(s, r) \rho(s) \int_{\alpha}^{\beta} q(s, \xi) d \mu(\xi)\right. \\
\quad-\frac{\rho(s)}{2 \sum_{i=1}^{m} \lambda_{i} M_{\theta} \sigma_{i}^{n-2}(s) \sigma_{i}^{\prime}(s)} \\
\quad \times\left[h_{1}(s, r)\right. \\
\left.\left.\quad+\sqrt{H(s, r)}\left(\frac{\rho^{\prime}(s)}{\rho(s)}-p(s)\right)\right]^{2}\right\} d s>0
\end{aligned}
$$

$\limsup _{t \rightarrow \infty} \int_{r}^{t}\left\{H(t, s) \rho(s) \int_{\alpha}^{\beta} q(s, \xi) d \mu(\xi)\right.$

$$
\begin{aligned}
& -\frac{\rho(s)}{2 \sum_{i=1}^{m} \lambda_{i} M_{\theta} \sigma_{i}^{n-2}(s) \sigma_{i}^{\prime}(s)} \\
& \times\left[h_{2}(t, s)\right. \\
& \left.\left.\quad-\sqrt{H(t, s)}\left(\frac{\rho^{\prime}(s)}{\rho(s)}-p(s)\right)\right]^{2}\right\} d s>0 .
\end{aligned}
$$

Then (1) is oscillatory.

Proof. For any $T \geq t_{0}$, let $a=T$. In (29), we choose $r=a$. Then there exists $c>a$ such that

$$
\int_{a}^{c}\left\{H(s, a) \rho(s) \int_{\alpha}^{\beta} q(s, \xi) d \mu(\xi)\right.
$$




$$
\begin{aligned}
& -\frac{\rho(s)}{2 \sum_{i=1}^{m} \lambda_{i} M_{\theta} \sigma_{i}^{n-2}(s) \sigma_{i}^{\prime}(s)} \\
& \left.\times\left[h_{1}(s, a)+\sqrt{H(s, a)}\left(\frac{\rho^{\prime}(s)}{\rho(s)}-p(s)\right)\right]^{2}\right\} d s>0 .
\end{aligned}
$$

In (30), we choose $r=c$, then there exists $b>c$ such that

$$
\begin{aligned}
& \int_{c}^{b}\left\{H(b, s) \rho(s) \int_{\alpha}^{\beta} q(s, \xi) d \mu(\xi)\right. \\
& \quad-\frac{\rho(s)}{2 \sum_{i=1}^{m} \lambda_{i} M_{\theta} \sigma_{i}^{n-2}(s) \sigma_{i}^{\prime}(s)} \\
& \left.\quad \times\left[h_{2}(b, s)-\sqrt{H(b, s)}\left(\frac{\rho^{\prime}(s)}{\rho(s)}-p(s)\right)\right]^{2}\right\} d s>0 .
\end{aligned}
$$

By dividing (31) and (32) by $H(c, a)$ and $H(b, c)$, respectively, and then adding them, we obtain (27). The conclusion thus comes from Theorem 7 . The proof is completed.

For the case of $H:=H(t-s) \in \Phi$, we have that $h_{1}(t-s)=h_{2}(t-s)$ and thus denote them by $h(t-s)$. The subclass of $\Phi$ containing such $H(t-s)$ is denoted by $\Phi_{0}$. Applying Theorem 7 to $\Phi_{0}$, and choosing $\rho=1$, we obtain the following.

Theorem 9. Assume that for each $T \geq t_{0}$ there exist $H \in \Phi_{0}$ and $a, c \in R$ such that $T \leq a<c$ and

$$
\begin{aligned}
& \int_{a}^{c} H(s-a)\left(\int_{\alpha}^{\beta}[q(s, \xi)+q(2 c-s, \xi)] d \mu(\xi)\right) d s \\
&>\frac{1}{2} \int_{a}^{c}\left\{\frac{[h(s-a)-p(s) \sqrt{H(s-a)}]^{2}}{\sum_{i=1}^{m} \lambda_{i} M_{\theta} \sigma_{i}^{n-2}(s) \sigma_{i}^{\prime}(s)}\right. \\
&\left.+\frac{[h(s-a)+p(2 c-s) \sqrt{H(s-a)}]^{2}}{\sum_{i=1}^{m} \lambda_{i} M_{\theta} \sigma_{i}^{n-2}(2 c-s) \sigma_{i}^{\prime}(2 c-s)}\right\} d s .
\end{aligned}
$$

Then (1) is oscillatory.

Proof. Let $b=2 c-a$. Then $H(b-c)=H(c-a)=H((b-a) / 2)$, and for any $\varphi \in L[a, b]$, we have

$$
\int_{c}^{b} \varphi(s) d s=\int_{a}^{c} \varphi(2 c-s) d s .
$$

Hence

$$
\begin{aligned}
& \int_{c}^{b} H(b-s)\left(\int_{\alpha}^{\beta} q(s, \xi) d \mu(\xi)\right) d s \\
& \quad=\int_{a}^{c} H(s-a)\left(\int_{\alpha}^{\beta} q(2 c-s, \xi) d \mu(\xi)\right) d s
\end{aligned}
$$

$$
\begin{gathered}
\int_{c}^{b} \frac{[h(b-s)+p(s) \sqrt{H(b-s)}]^{2}}{\sum_{i=1}^{m} \lambda_{i} M_{\theta} \sigma_{i}^{n-2}(s) \sigma_{i}^{\prime}(s)} d s \\
\quad=\int_{a}^{c} \frac{[h(s-a)+p(2 c-s) \sqrt{H(s-a)}]^{2}}{\sum_{i=1}^{m} \lambda_{i} M_{\theta} \sigma_{i}^{n-2}(2 c-s) \sigma_{i}^{\prime}(2 c-s)} d s .
\end{gathered}
$$

Thus (33) holds and implies that (27) holds for $H \in \Phi_{0}, \rho=$ 1 and therefore (1) is oscillatory by Theorem 7 . The proof is completed.

From the above oscillation criteria, we can obtain different sufficient conditions for oscillation of (1) by different choices of $H(t, s)$ and $\rho(s)$. For example, let

$$
H(t, s)=(t-s)^{\lambda}, \quad t \geq s \geq t_{0},
$$

where $\lambda>1$ is a constant. Then, $H \in \Phi_{0}$ and $h(t-s)=$ $\lambda(t-s)^{(\lambda / 2)-1}$. From Theorem 8, we have the following result.

Corollary 10. If there exists a function $\rho \in C^{\prime}\left(\left[t_{0}, \infty\right),(0, \infty)\right)$ and a constant $\lambda>1$ such that for each $r \geq t_{0}$,

$$
\begin{aligned}
\limsup _{t \rightarrow \infty} & \frac{1}{t^{\lambda-1}} \\
\times \int_{r}^{t}(s-r)^{\lambda} \rho(s) & \\
\quad \times\left\{\int_{\alpha}^{\beta} q(s, \xi) d \mu(\xi)\right. & \\
& -\frac{1}{2 \sum_{i=1}^{m} \lambda_{i} M_{\theta} \sigma_{i}^{n-2}(s) \sigma_{i}^{\prime}(s)} \\
& \left.\times\left[\frac{\lambda}{s-r}+\left(\frac{\rho^{\prime}(s)}{\rho(s)}-p(s)\right)\right]^{2}\right\} d s>0,
\end{aligned}
$$

$\limsup _{t \rightarrow \infty} \frac{1}{t^{\lambda-1}}$

$$
\begin{aligned}
& \times \int_{r}^{t}(t-s)^{\lambda} \rho(s) \\
& \times\left\{\int_{\alpha}^{\beta} q(s, \xi) d \mu(\xi)\right.
\end{aligned}
$$

$$
\begin{aligned}
& -\frac{1}{2 \sum_{i=1}^{m} \lambda_{i} M_{\theta} \sigma_{i}^{n-2}(s) \sigma_{i}^{\prime}(s)} \\
& \left.\times\left[\frac{\lambda}{t-s}-\left(\frac{\rho^{\prime}(s)}{\rho(s)}-p(s)\right)\right]^{2}\right\} d s>0 .
\end{aligned}
$$

Then (1) is oscillatory.

\section{Oscillation Results for $f\left(u_{1}, \ldots, u_{m}\right)$ without Monotonicity}

Throughout this section we assume that the following conditions hold: 
$\left(\mathrm{A}_{1}^{\prime}\right)$ there exists a function $\sigma(t) \in C^{\prime}\left(\left[t_{0}, \infty\right),(0, \infty)\right)$ such that $\sigma(t)=\min \left\{t, \min _{1 \leq i \leq m}\left\{\inf _{\xi \in[\alpha, \beta]} g_{i}(t, \xi)\right\}\right\}$, $\lim _{t \rightarrow \infty} \sigma(t)=\infty, \sigma^{\prime}(t)>0$.

$\left(\mathrm{A}_{2}^{\prime}\right)$ there exists a constant $\gamma>0$ and $i_{0} \in\{1,2, \ldots, m\}$ such that for sufficiently large $\left|u_{i}\right|\left(i \neq i_{0}\right)$

$$
\liminf _{\left|u_{i_{0}}\right| \rightarrow \infty}\left|\frac{f\left(u_{1}, \ldots, u_{m}\right)}{u_{i_{0}}}\right| \geq \gamma>0 .
$$

Lemma 11. Let $x(t)$ be an eventually positive solution of (1). Then, there exists a sufficiently large $T_{0} \geq t_{0}$ such that for $t \geq$ $T_{0}$, we have

$$
x^{\prime}(t)>0, \quad x^{(n-1)}(t)>0, \quad x^{(n)}(t) \leq 0 .
$$

The proof is similar to that of Lemma 4, thus we omit the details here.

Lemma 12. Let $x(t)$ be an eventually positive solution of (1). Then, there exists a sufficiently large $T_{0} \geq t_{0}$ such that for any interval $[c, b) \subset\left[T_{0}, \infty\right)$, if let

$$
u(t)=\frac{\rho(t) x^{(n-1)}(t)}{x[\sigma(t) / 2]}, \quad t \in[c, b),
$$

where $\rho(t) \in C^{\prime}\left(\left[t_{0}, \infty\right),(0, \infty)\right)$, then for any $H \in \Phi$,

$$
\begin{aligned}
\int_{c}^{b} \gamma H & (b, s) \rho(s)\left(\int_{\alpha}^{\beta} q(s, \xi) d \mu(\xi)\right) d s \\
\leq & H(b, c) u(c) \\
& +\frac{1}{2} \int_{c}^{b} \frac{\rho(s)}{M_{\theta} \sigma^{n-2}(s) \sigma^{\prime}(s)} \\
\times & {\left[h_{2}(b, s)-\sqrt{H(b, s)}\left(\frac{\rho^{\prime}(s)}{\rho(s)}-p(s)\right)\right]^{2} d s . }
\end{aligned}
$$

Proof. From (1) and (40) we have that for $t \in[c, b)$

$$
\begin{aligned}
u^{\prime}(t) & \frac{\rho(t) x^{(n)}(t)+\rho^{\prime}(t) x^{(n-1)}(t)}{x[\sigma(t) / 2]} \\
& -\frac{u(t)}{2 x[\sigma(t) / 2]} x^{\prime}\left[\frac{\sigma(t)}{2}\right] \sigma^{\prime}(t) \\
= & -\rho(t) \frac{\int_{\alpha}^{\beta} q(t, \xi) f\left(x\left[g_{1}(t, \xi)\right], \ldots, x\left[g_{m}(t, \xi)\right]\right) d \mu(\xi)}{x[\sigma(t) / 2]} \\
& +\left(\frac{\rho^{\prime}(t)}{\rho(t)}-p(t)\right) u(t)-\frac{x^{\prime}[\sigma(t) / 2]}{2 x[\sigma(t) / 2]} \sigma^{\prime}(t) u(t) .
\end{aligned}
$$

From Lemma 11, there exists a sufficiently large $T_{0} \geq t_{0}$ such that for all $t \geq T_{0}$ (39) hold and further from $\left(\mathrm{A}_{1}^{\prime}\right)$

$$
\begin{array}{r}
\frac{\sigma(t)}{2} \leq \sigma(t) \leq t, \quad g_{i}(t, \xi) \geq \sigma(t) \geq \frac{\sigma(t)}{2}, \\
i=1,2, \ldots, m ; \xi \in[\alpha, \beta] .
\end{array}
$$

Hence, we have for all $t \geq T_{0}$,

$$
\begin{array}{r}
x^{(n-1)}\left[\frac{\sigma(t)}{2}\right] \geq x^{(n-1)}(t), \quad x\left[g_{i}(t, \xi)\right] \geq x[\sigma(t) / 2], \\
i=1,2, \ldots, m ; \xi \in[\alpha, \beta] .
\end{array}
$$

From (44) and $\left(\mathrm{A}_{2}^{\prime}\right)$, for all $t \geq T_{0}$

$$
\begin{aligned}
& f\left(x\left[g_{1}(t, \xi)\right], \ldots, x\left[g_{m}(t, \xi)\right]\right) \\
& \quad \geq \gamma x\left[g_{i_{0}}(t, \xi)\right] \geq \gamma x\left[\frac{\sigma(t)}{2}\right], \quad \xi \in[\alpha, \beta] .
\end{aligned}
$$

Thus, for all $t \geq T_{0}$

$$
\begin{aligned}
& \frac{\int_{\alpha}^{\beta} q(t, \xi) f\left(x\left[g_{1}(t, \xi)\right], \ldots, x\left[g_{m}(t, \xi)\right]\right) d \mu(\xi)}{x[\sigma(t) / 2]} \\
& \quad \geq \gamma \int_{\alpha}^{\beta} q(t, \xi) d \mu(\xi) .
\end{aligned}
$$

Therefore, from (42)-(46) and Lemma 3, we obtain

$$
\begin{aligned}
u^{\prime}(t) \leq & -\gamma \rho(t) \int_{\alpha}^{\beta} q(t, \xi) d \mu(\xi) \\
& +\left(\frac{\rho^{\prime}(t)}{\rho(t)}-p(t)\right) u(t) \\
& -\frac{1}{2} \rho^{-1}(t) M_{\theta} \sigma^{n-2}(t) \sigma^{\prime}(t) u^{2}(t) .
\end{aligned}
$$

The rest of the proof is similar to that of Lemma 5 and thus we omit the details here.

Similar to the proof in Section 2, we have the following results.

Lemma 13. Let $x(t)$ be an eventually positive solution of (1). Then, there exists a sufficiently large $T_{0} \geq t_{0}$ such that, for any interval $(a, c] \subset\left[T_{0}, \infty\right)$, if let $u(t)$ be defined by $(40)$ on $(a, c]$, then for any $H \in \Phi$,

$$
\begin{aligned}
\int_{a}^{c} \gamma H(s, a) \rho(s)\left(\int_{\alpha}^{\beta} q(s, \xi) d \mu(\xi)\right) d s \leq-H(c, a) u(c) \\
+\frac{1}{2} \int_{a}^{c} \frac{\rho(s)}{M_{\theta} \sigma^{n-2}(s) \sigma^{\prime}(s)} \\
\times\left[h_{1}(s, a)+\sqrt{H(s, a)}\left(\frac{\rho^{\prime}(s)}{\rho(s)}-p(s)\right)\right]^{2} d s .
\end{aligned}
$$

The following theorem is an immediate result from Lemmas 12 and 13. 
Theorem 14. Assume that for each $T \geq t_{0}$ there exist $H \in \Phi$, $\rho \in C^{\prime}\left(\left[t_{0}, \infty\right),(0, \infty)\right)$ and $a, b, c \in R$, such that $T \leq a<c<$ $b$ and

$$
\begin{aligned}
\frac{1}{H(c, a)} \int_{a}^{c} & \gamma H(s, a) \rho(s)\left(\int_{\alpha}^{\beta} q(s, \xi) d \mu(\xi)\right) d s \\
+ & \frac{1}{H(b, c)} \int_{c}^{b} \gamma H(b, s) \rho(s)\left(\int_{\alpha}^{\beta} q(s, \xi) d \mu(\xi)\right) d s \\
>\frac{1}{2 M_{\theta}} & \left\{\frac{1}{H(c, a)} \int_{a}^{c} \frac{\rho(s)}{\sigma^{n-2}(s) \sigma^{\prime}(s)}\right. \\
& \times\left[h_{1}(s, a)+\sqrt{H(s, a)}\left(\frac{\rho^{\prime}(s)}{\rho(s)}-p(s)\right)\right]^{2} d s \\
& +\frac{1}{H(b, c)} \int_{c}^{b} \frac{\rho(s)}{\sigma^{n-2}(s) \sigma^{\prime}(s)} \\
& \left.\times\left[h_{2}(b, s)-\sqrt{H(b, s)}\left(\frac{\rho^{\prime}(s)}{\rho(s)}-p(s)\right)\right]^{2} d s\right\} .
\end{aligned}
$$

Then (1) is oscillatory.

Theorem 15. Assume that for some $H \in \Phi$ and $\rho \in$ $C^{\prime}\left(\left[t_{0}, \infty\right),(0, \infty)\right)$, and for each $r \geq t_{0}$,

$$
\begin{aligned}
& \limsup _{t \rightarrow \infty} \int_{r}^{t}\left\{\gamma H(s, r) \rho(s) \int_{\alpha}^{\beta} q(s, \xi) d \mu(\xi)\right. \\
&- \frac{\rho(s)}{2 M_{\theta} \sigma^{n-2}(s) \sigma^{\prime}(s)} \\
& \times\left[h_{1}(s, r)\right. \\
&\left.\left.+\sqrt{H(s, r)}\left(\frac{\rho^{\prime}(s)}{\rho(s)}-p(s)\right)\right]^{2}\right\} d s>0,
\end{aligned}
$$

$$
\begin{aligned}
\limsup _{t \rightarrow \infty} \int_{r}^{t}\left\{\gamma H(t, s) \rho(s) \int_{\alpha}^{\beta} q(s, \xi) d \mu(\xi)\right. \\
-\quad \frac{\rho(s)}{2 M_{\theta} \sigma^{n-2}(s) \sigma^{\prime}(s)} \\
\quad \times\left[h_{2}(t, s)\right. \\
\left.\left.\quad-\sqrt{H(t, s)}\left(\frac{\rho^{\prime}(s)}{\rho(s)}-p(s)\right)\right]^{2}\right\} d s>0 .
\end{aligned}
$$

Then (1) is oscillatory.
Theorem 16. Assume that for each $T \geq t_{0}$, there exist $H \in \Phi_{0}$ and $a, c \in R$ such that $T \leq a<c$ and

$$
\begin{gathered}
\int_{a}^{c} \gamma H(s-a)\left(\int_{\alpha}^{\beta}[q(s, \xi)+q(2 c-s, \xi)] d \mu(\xi)\right) d s \\
>\frac{1}{2 M_{\theta}} \int_{a}^{c}\left\{\frac{[h(s-a)-p(s) \sqrt{H(s-a)}]^{2}}{\sigma^{n-2}(s) \sigma^{\prime}(s)}\right. \\
\left.+\frac{[h(s-a)+p(2 c-s) \sqrt{H(s-a)}]^{2}}{\sigma^{n-2}(2 c-s) \sigma^{\prime}(2 c-s)}\right\} d s .
\end{gathered}
$$

Then (1) is oscillatory.

Corollary 17. If there exists a function $\rho \in C^{\prime}\left(\left[t_{0}, \infty\right),(0, \infty)\right)$ and a constant $\lambda>1$ such that for each $r \geq t_{0}$, the following two inequalities hold

$$
\begin{aligned}
\limsup _{t \rightarrow \infty} & \frac{1}{t^{\lambda-1}} \int_{r}^{t} \gamma(s-r)^{\lambda} \rho(s) \\
\times & \left\{\int_{\alpha}^{\beta} q(s, \xi) d \mu(\xi)-\frac{1}{2 M_{\theta} \sigma^{n-2}(s) \sigma^{\prime}(s)}\right. \\
& \left.\times\left[\frac{\lambda}{s-r}+\left(\frac{\rho^{\prime}(s)}{\rho(s)}-p(s)\right)\right]^{2}\right\} d s>0, \\
\limsup _{t \rightarrow \infty} \frac{1}{t^{\lambda-1}} \int_{r}^{t} \gamma(t-s)^{\lambda} \rho(s) & \\
\times & \left\{\int_{\alpha}^{\beta} q(s, \xi) d \mu(\xi)-\frac{1}{2 M_{\theta} \sigma^{n-2}(s) \sigma^{\prime}(s)}\right. \\
\times & {\left.\left[\frac{\lambda}{t-s}-\left(\frac{\rho^{\prime}(s)}{\rho(s)}-p(s)\right)\right]^{2}\right\} d s>0 . }
\end{aligned}
$$

Then (1) is oscillatory.

\section{Examples}

In this section we demonstrate the applications of our oscillation criteria through two examples. We will see that the equations in the examples are oscillatory based on the results in Sections 2 and 3.

Example 1. Consider the following nonlinear damped differential equation:

$$
\begin{aligned}
x^{(4)}(t)+ & \frac{2 t}{\exp \left(t^{2}\right)} x^{(3)}(t) \\
+ & \int_{0}^{1} e^{2 t+\xi}\left[x(t+\xi)+x\left(3 t+\xi^{2}\right)\right. \\
& \left.+x^{3}(t+\xi)+x^{5}\left(3 t+\xi^{2}\right)\right] d \xi=0,
\end{aligned}
$$


where $t \geq 1, p(t)=\left(2 t / \exp \left(t^{2}\right)\right), q(t, \xi)=e^{2 t+\xi}, f\left(u_{1}, u_{2}\right)=$ $u_{1}+u_{2}+u_{1}^{3}+u_{2}^{5}, g_{1}(t, \xi)=t+\xi, g_{2}(t, \xi)=3 t+\xi^{2}, \mu(\xi)=\xi$. It is clear that for $t_{1} \geq 1$

$$
\begin{gathered}
\lim _{t \rightarrow \infty} \int_{t_{1}}^{t} \exp \left(-\int_{t_{1}}^{s} p(\tau) d \tau\right) d s \\
=\lim _{t \rightarrow \infty} \int_{t_{1}}^{t} \exp \left(-\int_{t_{1}}^{s} \frac{2 \tau}{\exp \left(\tau^{2}\right)} d \tau\right) d s=\infty \\
\sigma_{1}(t)=t, \quad \sigma_{2}(t)=t \\
\frac{\partial f}{\partial u_{1}}=1+3 u_{1}^{2} \geq 1=\lambda_{1} \\
\frac{\partial f}{\partial u_{2}}=1+5 u_{2}^{4} \geq 1=\lambda_{2} .
\end{gathered}
$$

Applying Corollary 10 with $\lambda=2$ and $\rho(s)=s^{3}$, we have through a straightforward computation that

$$
\begin{aligned}
\limsup _{t \rightarrow \infty} & \frac{1}{t^{\lambda-1}} \int_{r}^{t}(s-r)^{\lambda} \rho(s) \\
& \times\left\{\int_{\alpha}^{\beta} q(s, \xi) d \mu(\xi)-\frac{1}{2 \sum_{i=1}^{m} \lambda_{i} M_{\theta} \sigma_{i}^{n-2}(s) \sigma_{i}^{\prime}(s)}\right. \\
& \left.\times\left[\frac{\lambda}{s-r}+\left(\frac{\rho^{\prime}(s)}{\rho(s)}-p(s)\right)\right]^{2}\right\} d s \\
=\limsup _{t \rightarrow \infty} \frac{1}{t} \int_{r}^{t}(s-r)^{2} s^{3} & \times\left\{\int_{0}^{1} e^{2 s+\xi} d \xi-\frac{1}{4 M_{\theta} s^{2}}\left[\frac{5 s-3 r}{s(s-r)}\right.\right. \\
& \left.\left.-\frac{2 s}{\exp \left(s^{2}\right)}\right]^{2}\right\} d s=\infty
\end{aligned}
$$$$
\limsup _{t \rightarrow \infty} \frac{1}{t^{\lambda-1}}
$$$$
\times \int_{r}^{t}(t-s)^{\lambda} \rho(s)
$$$$
\times\left\{\int_{\alpha}^{\beta} q(s, \xi) d \mu(\xi)\right.
$$$$
-\frac{1}{2 \sum_{i=1}^{m} \lambda_{i} M_{\theta} \sigma_{i}^{n-2}(s) \sigma_{i}^{\prime}(s)}
$$$$
\left.\times\left[\frac{\lambda}{t-s}+\left(\frac{\rho^{\prime}(s)}{\rho(s)}-p(s)\right)\right]^{2}\right\} d s
$$$$
=\limsup _{t \rightarrow \infty} \frac{1}{t}
$$$$
\times \int_{r}^{t}(t-s)^{2} s^{3}
$$

$$
\begin{aligned}
& \times\left\{\int_{0}^{1} e^{2 s+\xi} d \xi\right. \\
& \left.\quad-\frac{1}{4 M_{\theta} s^{2}}\left[\frac{5 s-3 t}{s(t-s)}+\frac{2 s}{\exp \left(s^{2}\right)}\right]^{2}\right\} \\
& \times d s=\infty .
\end{aligned}
$$

Therefore (37) hold and we conclude by Corollary 10 that (55) is oscillatory.

Example 2. Consider the following nonlinear damped differential equation:

$$
\begin{array}{r}
x^{(4)}(t)+\exp (-t) x^{(3)}(t) \\
+\int_{0}^{\pi / 2} \frac{t^{2} \sin 2 \xi}{1+\sin ^{2} \xi} \frac{x(t+\sin \xi)}{2-\exp \left(-x^{2}(t+\cos \xi)\right)} d \xi=0, \\
t \geq 1,
\end{array}
$$

where $p(t)=1 / e^{t}, q(t, \xi)=t^{2} \sin 2 \xi /\left(1+\sin ^{2} \xi\right), \quad f\left(u_{1}, u_{2}\right)=$ $u_{2} /\left(2-\exp \left(-u_{1}^{2}\right)\right), g_{1}(t, \xi)=t+\cos \xi, g_{2}(t, \xi)=t+$ $\sin \xi, \mu(\xi)=\xi$. In this example,

$$
\frac{\partial f}{\partial u_{1}}=-\frac{2 u_{1} u_{2} \exp \left(-u_{1}^{2}\right)}{\left(2-\exp \left(-u_{1}^{2}\right)\right)^{2}} .
$$

Clearly, Corollary 10 does not apply to (58). However, with $\lambda=2$ and $\rho(t)=1$, we can prove the oscillatory character of (58) by Corollary 17. Noting that

$$
\begin{gathered}
\frac{f\left(u_{1}, u_{2}\right)}{u_{2}}=\frac{1}{2-\exp \left(-u_{1}^{2}\right)} \geq \frac{1}{2}=\gamma, \quad \forall u_{2} \neq 0, \\
\lim _{t \rightarrow \infty} \int_{t_{1}}^{t} \exp \left(-\int_{t_{1}}^{s} p(\tau) d \tau\right) d s \\
\quad=\lim _{t \rightarrow \infty} \int_{t_{1}}^{t} \exp \left(-\int_{t_{1}}^{s} \frac{1}{e^{\tau}} d \tau\right) d s=\infty,
\end{gathered}
$$

for $t_{1} \geq 1$ and $\sigma(t)=t$, we have

$$
\begin{aligned}
& \limsup _{t \rightarrow \infty} \frac{1}{t^{\lambda-1}} \\
& \times \int_{r}^{t} \gamma(s-r)^{\lambda} \rho(s) \\
& \times\left\{\int_{\alpha}^{\beta} q(s, \xi) d \mu(\xi)-\frac{1}{2 M_{\theta} \sigma^{n-2}(s) \sigma^{\prime}(s)}\right. \\
&\left.\times\left[\frac{\lambda}{s-r}+\left(\frac{\rho^{\prime}(s)}{\rho(s)}-p(s)\right)\right]^{2}\right\} d s
\end{aligned}
$$




$$
\begin{aligned}
& =\limsup _{t \rightarrow \infty} \frac{1}{t} \int_{r}^{t} \frac{1}{2}(s-r)^{2} \\
& \times\left\{\int_{0}^{\pi / 2} \frac{s^{2} \sin 2 \xi}{1+\sin ^{2} \xi} d \xi\right. \\
& \left.-\frac{1}{2 M_{\theta} s^{2}}\left[\frac{2}{s-r}-\frac{1}{e^{s}}\right]^{2}\right\} d s \\
& =\limsup _{t \rightarrow \infty} \frac{1}{t} \int_{r}^{t}\left\{\frac{1}{2} \ln 2 s^{2}(s-r)^{2}\right. \\
& \left.-\frac{\left(2 e^{s}-s+r\right)^{2}}{4 M_{\theta} s^{2} e^{2 s}}\right\} d s=\infty \\
& \limsup _{t \rightarrow \infty} \frac{1}{t^{\lambda-1}} \int_{r}^{t} \gamma(t-s)^{\lambda} \rho(s) \\
& \times\left\{\int_{\alpha}^{\beta} q(s, \xi) d \mu(\xi)\right. \\
& -\frac{1}{2 M_{\theta} \sigma^{n-2}(s) \sigma^{\prime}(s)} \\
& \left.\times\left[\frac{\lambda}{t-s}-\left(\frac{\rho^{\prime}(s)}{\rho(s)}-p(s)\right)\right]^{2}\right\} d s=\infty,
\end{aligned}
$$

therefore (53) and (54) hold and we conclude by Corollary 10 that $(58)$ is oscillatory.

\section{Conflict of Interests}

The authors declare that they have no conflict of interests.

\section{Authors' Contribution}

All authors completed the paper together. All authors read and approved the final paper.

\section{Acknowledgments}

This paper is supported by the National Natural Science Foundation of China (11271106), the Natural Science Foundation of Hebei Province of China (A2013201232), and the Science and Technology Project of Baoding of China (13ZG022).

\section{References}

[1] B. Ayanlar and A. Tiryaki, "Oscillation theorems for nonlinear second order differential equations with damping," Acta Mathematica Hungarica, vol. 89, no. 1-2, pp. 1-13, 2000.

[2] W.-T. Li and R. P. Agarwal, "Interval oscillation criteria for second order forced nonlinear differential equations with damping," Panamerican Mathematical Journal, vol. 11, no. 3, pp. 109-117, 2001.
[3] W.-T. Li and R. P. Agarwal, "Interval oscillation criteria for second-order nonlinear differential equations with damping," Computers \& Mathematics with Applications, vol. 40, no. 2-3, pp. 217-230, 2000.

[4] M. Kirane and Y. V. Rogovchenko, "Oscillation results for a second order damped differential equation with nonmonotonous nonlinearity," Journal of Mathematical Analysis and Applications, vol. 250, no. 1, pp. 118-138, 2000.

[5] P. Wang and Y. Wu, "Oscillation of certain second-order functional differential equations with damping," Journal of Computational and Applied Mathematics, vol. 157, no. 1, pp. 4956, 2003.

[6] Y. G. Sun, "Oscillation of second order functional differential equations with damping," Applied Mathematics and Computation, vol. 178, no. 2, pp. 519-526, 2006.

[7] P. Wang and M. Wu, "Oscillation of certain second order nonlinear damped difference equations with continuous variable," Applied Mathematics Letters, vol. 20, no. 6, pp. 637-644, 2007.

[8] Z. Xu, "Oscillation theorems for damped elliptic differential equations of second order," Hiroshima Mathematical Journal, vol. 38, no. 1, pp. 1-17, 2008.

[9] E. Tun and H. Avc, "New oscillation theorems for a class of second-order damped nonlinear differential equations," Ukrainian Mathematical Journal, vol. 63, no. 9, pp. 1441-1457, 2012.

[10] I. T. Kiguradze, "On the oscillatory character of solutions of the equation $d^{m} u / d t^{m}+a(t)|u|^{n}$ sgnu $=0$," vol. 65, pp. 172-187, 1964.

[11] C. H. G. Philos, "A new criterion for the oscillatory and asymptonic behavior of delay differential equations," Bulletin de L'Académie Polonaise des Science, vol. 39, pp. 61-64, 1981. 


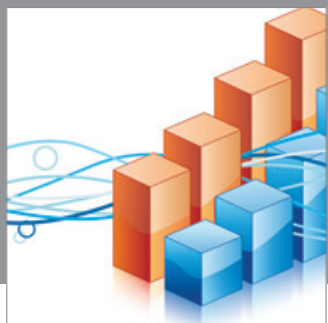

Advances in

Operations Research

mansans

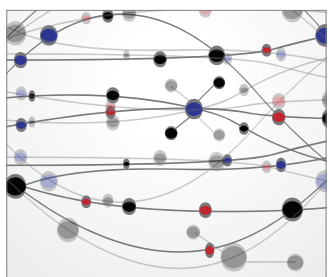

The Scientific World Journal
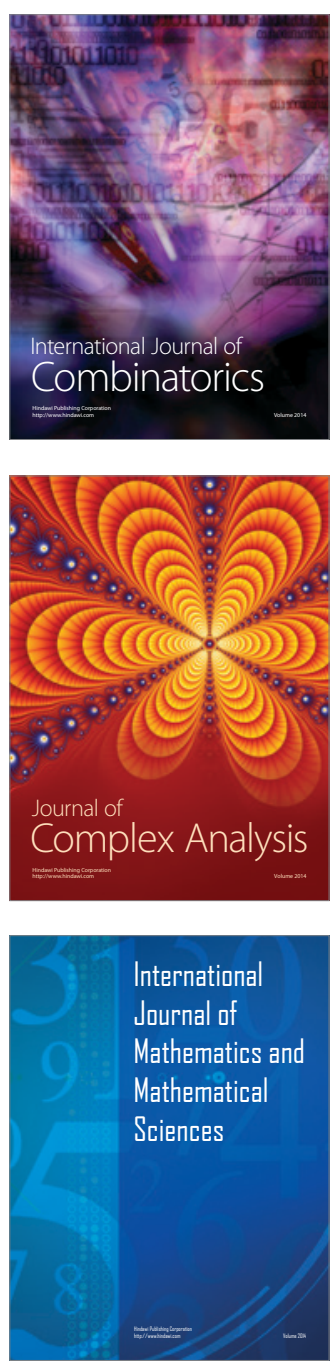
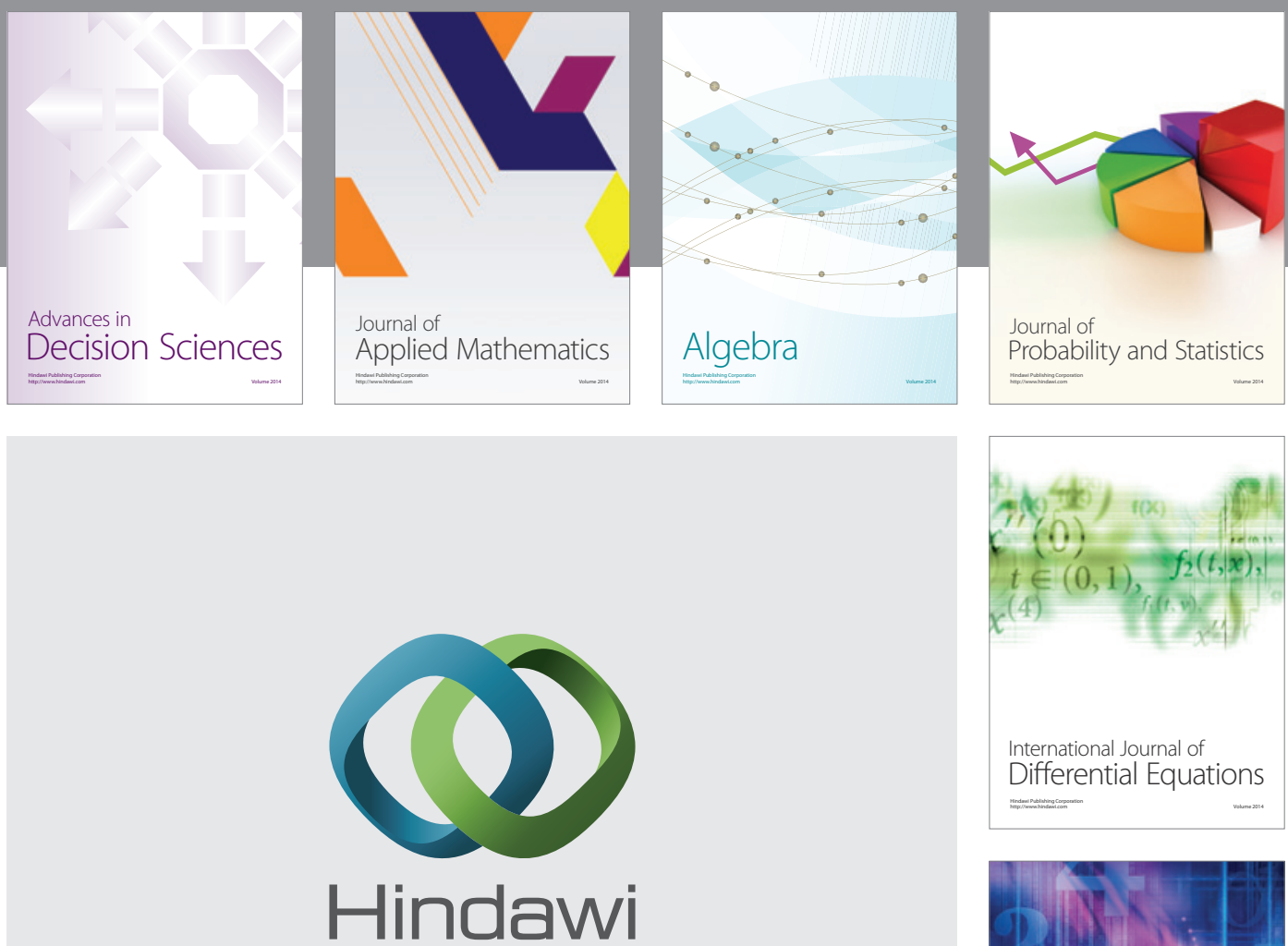

Submit your manuscripts at http://www.hindawi.com
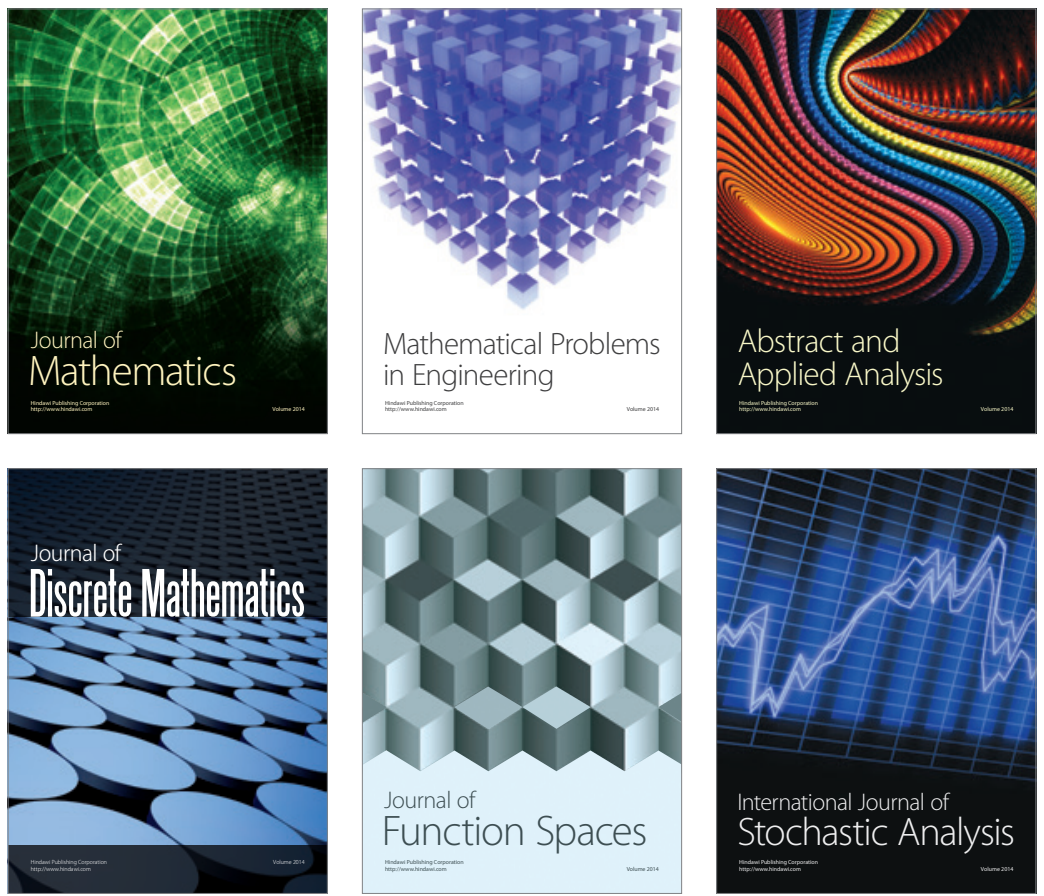

Journal of

Function Spaces

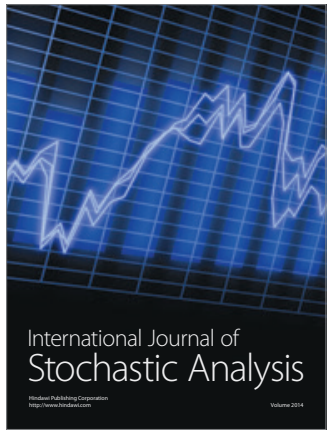

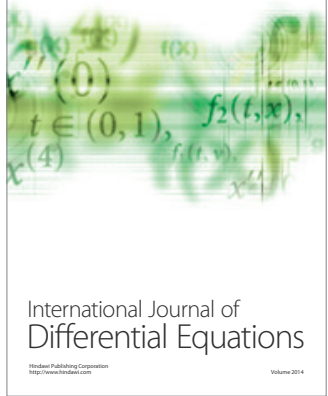
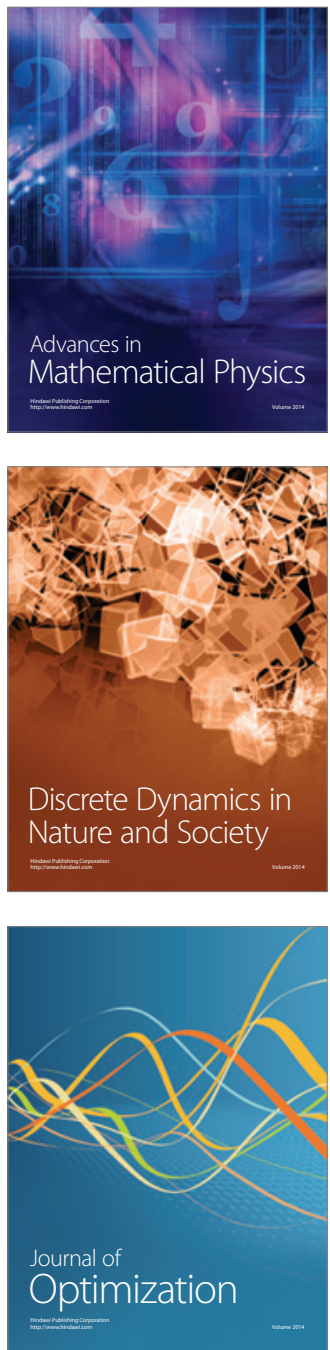